\title{
Comparing the Renal Profile of Patients with Dementia Admitted to a Psychiatric Unit to Those with Delirium/Dementia Seen by the Liaison Psychiatric Services in District General Hospital (DGH) In Wales
}

\author{
Aziz VM ${ }^{1 *}$, Sullivan $\mathrm{G}^{2}$ and Roufael $\mathrm{R}^{3}$ \\ ${ }^{1}$ Department of Psychiatrist, Devon Partnership NHS Trust, UK \\ ${ }^{2}$ Department of Psychiatrist, South Wales, UK \\ ${ }^{3}$ Specialty Registrar, All Wales Higher Training Psychiatric Rotation, UK
}

*Corresponding author: Aziz VM, Department of Psychiatrist, Devon Partnership NHS Trust, UK

Received Date: May 11, 2019

Published Date: May 28, 2019

\section{Abstract}

In this paper we aimed at looking at the renal profile and its impact on cognition. The research aims to test the renal profile of patients with dementia or delirium as part of the physical health care check and to review its effect on cognition. We looked at renal profile for all patients admitted to a psychiatric unit with a diagnosis of dementia and compared them with patients diagnosed with delirium/ dementia who were referred to the psychiatric liaison team within 6 months period.

Seventy-six patients suffering dementia were admitted to a Psychiatric Unit and sixty-one suffering delirium/ dementia seen by Liaison Psychiatry Services in District General Hospitals (DGH) were sampled (Total 137 patients). The mean age of the sample was 80.85 years old (SD = 8.32) and $56.9 \%$ were females $(n=78)$. Vascular risk factors were present in $79 \%$ of dementia patients in the psychiatric unit $(n=60)$ versus $83.6 \%$ of Liaison psychiatry patients $(n=51)$. CT head scan were done on 129 patients $(94 \%)$, and $69 \%(n=89)$ of the scans showed vascular brain insult. Multivariate analysis yielded no significant association between both the diagnosis and the presence of vascular risk factors in both groups and the renal profile.

Our paper highlighted the need to ensure that cognitive testing and brain scans are completed for all patients as much as possible. Both Mental Health Services and District General Hospitals are to consider implementing a dementia-physical health pathway to improve the physical health of patients with cognitive impairment. Comparing the Renal Profile of patients with Dementia admitted to a Psychiatric Unit to those with Delirium/ Dementia seen by the Liaison Psychiatric Services in District General Hospital (DGH) in Wales.

Keywords: Renal; Profile; Impairment; Dementia; Delirium; Vascular; Cognitive

\section{Introduction}

Physiological functions, including kidney function, are strongly associated with age. Glomerular filtration rate (GFR), which is a marker of chronic kidney disease (CKD), declines with age in healthy adults [1]. CKD is defined as a condition with an estimated GFR (eGFR) of less than $60 \mathrm{~mL} / \mathrm{min}$ per $1.73 \mathrm{~m} 2$ or continuous presence of kidney impairment (e.g., albuminuria) for 3 months or longer [2].
The Hisayama Study Group has investigated the relationship between CKD and vascular risk and reported that the former was an important risk factor for cardiovascular disease [3] and that people with metabolic syndrome had a higher incidence of CKD [4]. CKD has also been established as an independent risk factor for cerebrovascular disease (CVD) and cardiovascular disease [5,6]. One study [5] reported that, in middle-aged community-based 
adults, the combination of CKD and anaemia was associated with a substantially greater risk of stroke independent of other known risk factors for stroke.

The prevalence of CKD has been shown to be higher in older adults in Japan [7] and internationally [8]. Approximately $20 \%$ of the Japanese adult population is estimated to have Stage 3 to 5 CKD, which represents kidney impairment [7]. Recent studies have shown impaired kidney function to be associated with greater prevalence of cognitive impairment $[9,10]$ a more-rapid rate of cognitive decline [11], and incident cognitive impairment [12-14]. Three studies reported associations between kidney function and cognitive impairment at an eGFR threshold of less than $60 \mathrm{~mL} / \mathrm{min}$ per $1.73 \mathrm{~m} 11,13$ a fourth reported that the threshold effect was at a lower GFR (eGFR<45 mL/min per $1.73 \mathrm{~m} 2)$ [14]. and a fifth reported the threshold effect to be at a much lower GFR $($ GFR $<30$ $\mathrm{mL} / \mathrm{min}$ per $1.73 \mathrm{~m} 2$ ) [10]. All studies reported age, hypertension, and diabetes mellitus to be independent factors in the association between kidney function and cognition, but an association between CKD and dementia onset has not been reported, with the exception of one study [12], which used serum creatinine but not eGFR for assessment of kidney function.

In this research we aimed at looking at the renal profile and its impact on cognition. The paper aims to test the renal profile of patients with dementia or delirium as part of the physical health care check and to review its effect on cognition.

\section{Methodology}

Our design was a combined cross sectional and prospective data analysis. We looked at renal profile for all patients admitted to a psychiatric unit with a diagnosis of dementia and compared them with patients diagnosed with delirium/ dementia who were referred to the psychiatric liaison team within a 6 months' period.

A clinical team consisting of an inpatient consultant psychiatrist, a liaison consultant psychiatrist and higher trainee registrar reviewed the medical records, ensured the renal profile was completed, and did cognitive assessment using either he Addenbrooke's Cognitive Examination-III (ACE-III) which is a brief cognitive test that assesses five cognitive domains: attention, memory, verbal fluency, language and visuospatial abilities; or Montreal Cognitive Assessment (MoCA) together with reviewing the brain scans where indicated. The patient's diagnosis, medical history, co-morbidities and medications were also noted together with any relevant demographic data. The vascular risk factors such as CKD, hypertension, dyslipidaemia, diabetes mellitus, IHD, and anaemia were noted.

\section{Result}

\section{Demographics (Table 1)}

Seventy-six patients suffering dementia were admitted to a Psychiatric Unit and sixty-one suffering delirium/ dementia seen by Liaison Psychiatry Services in District General Hospitals (DGH) were sampled (Total 137 patients). The mean age of the sample was 80.85 years old $(S D=8.32)$ and $56.9 \%$ were females $(n=78)$. Vascular risk factors were present in $79 \%$ of dementia patients in the psychiatric unit $(n=60)$ versus $83.6 \%$ of Liaison psychiatry patients $(n=51)$.

$31.5 \%(n=24)$ of patients in the psychiatric unit while $27.9 \%$ $(n=17)$ of DGH patients had positive MSU with E-Coli, E Faecalis, Proteus or mixed heavy growth. Females were associated with more urine infections than males, X2 $(1, \mathrm{~N}=76)=10.316, \mathrm{p}=.001$. Odd ratio (OR) for females $=0.44$ vs. OR for males $=0.18$. CT head scans were done in 129 patients (94\%), and 69\% (n= 89) of the scans showed vascular brain insult. Cognitive testing was done in 40 patients with dementia in the psychiatric ward $(52.6 \%$ ACE3 or MOCA) and 59 patients with delirium/ dementia in the DGH $(96.7 \%$ had MOCA). The mean number of comorbidities per individual was 4 items $(\mathrm{SD} \pm 2.03)$. While the mean number of medications was 10.3 items (SD \pm 4 , range 4-18) (Table 1$)$.

Table 1: Demographics.

\begin{tabular}{|c|c|c|}
\hline Demographics & Dementia (N= 76) & Liaison Psychiatry (N= 61) \\
\hline Age (mean \pm SD) & $78.03 \pm 8.3$ & $84.4 \pm 6.9$ \\
\hline Gender F \% (N) & $56.6 \%(43)$ & $57.4 \%(35)$ \\
\hline $\begin{array}{c}\text { Vascular risk } \\
\text { factors \% (N) }\end{array}$ & $79 \%(60)$ & $83.6 \%(51)$ \\
\hline Positive MSU \% (N) & $31.5 \%(24)$ & $27.9 \%(17)$ \\
\hline $\begin{array}{c}\text { CT head scan } \\
\text { completed \% (N) }\end{array}$ & $94.7 \%(72)$ & $93.4 \%(57)$ \\
\hline $\begin{array}{c}\text { Cognitive testing \% } \\
\text { (ACE3 or MOCA) }\end{array}$ & $52.6 \%(40)$ & $96.7 \%(59)$ \\
\hline
\end{tabular}

\section{Diagnosis}

Vascular dementia and Alzheimer's constituted the majority of diagnoses (72.3\%). Alzheimer's disease $(37.2 \%, \mathrm{n}=51)$, Vascular dementia $(35 \%, n=48)$, mixed dementia $(11 \%, n=15)$, Lewy Body dementia (5\%), and other diagnoses included Parkinson's disease, Alcohol-related brain damage, Frontotemporal dementia, and Dementia NOS, and in six patients the primary diagnosis was delirium (in the DGH).

\section{Co-morbidities}

Hypercholesterolemia, Hypertension, AF, COPD, Osteoarthritis, Macular degeneration, Myocardial infarction, IDDM, CKD, Breast cancer, Uterine cancer, Stoke, DVT, Type 2 Diabetes Mellitus, Recurrent UTIs, Inguinal hernia, Heavy smoking, DVT, Ischaemic heart disease, Hypothyroidism, TIA, Parkinson's disease, Angina, Asthma, Epilepsy, Rectal cancer, Arthritis, Alcohol excess, Myasthenia gravis, OA Knees, CCF, Pernicious anaemia, Hiatus hernia, Vertigo, Osteoporosis, Carotid artery disease, Hip fractures, Cervical spondylosis, Constipation, rheumatic fever, systematic focal epilepsy, Lung cancer, spondylosis, hysterectomy, Inguinal hernia, osteoarthritis, Grand mal seizures, Duodenal ulcer, Diverticulitis, CVA, Hydronephrosis, impaired glucose tolerance, cataracts, Retinal detachment, Colon cancer/subtotal colectomy, lower urinary tract symptoms, fractured neck of femur, Prostate cancer, spinal stenosis, reflux oesophagitis, varicose veins. 
Archoidosis, Kidney disease, DVT/PE, Urinary retention, Haemorrhoids, Myoclonic jerks, Progressive supranuclear palsy, overactive bladder, benign prostatic hypertrophy, Knee surgery, Depression, Chronic Kidney Disease, Viral encephalitis, haematuria, bilateral leg oedema, pressure sores, thyrotoxicosis and sepsis, chronic back pain, renal calculi, left posterior vitreous reattachment, hypotension, cholecystectomy, chronic kidney disease stage 4, IBS, anxiety, spondylitis, palpitations, acquired brain injury secondary car accident, Pseudo gout, Falls, Cholecystectomy, fractured wrist, SDH, Non-toxic multinodular goiter (Table 2).

Table 2: Showed the results of the renal profile. T-test showed significant difference in between the two groups in relation to sodium (higher levels in group 1), creatinine (higher levels in group 2) and eGFR (lower levels in group 2).

\begin{tabular}{|c|c|c|c|}
\hline Table 2 & $\begin{array}{c}\text { Dementia } \\
\text { (group1) } \\
\text { (Mean } \pm \text { SD) }\end{array}$ & $\begin{array}{c}\text { Liaison } \\
\text { Psychiatry } \\
\text { (group 2) } \\
\text { (Mean } \pm \text { SD) }\end{array}$ & T-Test Significance \\
\hline Sodium & $141.55 \pm 3.85$ & $138.34 \pm 5.66$ & $\mathrm{t}=4.201, \mathrm{P}=0.000$ \\
\hline Potassium & $4.29 \pm 0.46$ & $4.67 \pm 0.62$ & $\mathrm{t}=-.887, \mathrm{P}=0.379$ \\
\hline Urea & $7.7 \pm 6.69$ & $8.53 \pm 4.08$ & $\mathrm{t}=-.635, \mathrm{P}=0.528$ \\
\hline Creatinine & $84.43 \pm 23.97$ & $103.25 \pm 54.16$ & $\mathrm{t}=-3.045, \mathrm{P}=0.003$ \\
\hline eGFR & $68.71 \pm 22.30$ & $57.12 \pm 19.77$ & $\mathrm{t}=-1.036, \mathrm{P}=0.000$ \\
\hline Glucose & $7.1 \pm 2.00$ & $6.8 \pm 1.98$ & $\mathrm{t}=4.201, \mathrm{P}=0.305$ \\
\hline
\end{tabular}

Table 3: Multivariate analysis yielded no significant association between both the diagnosis and the presence of vascular risk factors in both groups and the renal profile. There was no significant interaction between diagnosis and vascular risk factors.

\begin{tabular}{|c|c|c|c|c|c|}
\hline \multirow{2}{*}{$\begin{array}{c}\text { Tests of } \\
\text { Between- } \\
\text { Subjects } \\
\text { Effects }\end{array}$} & \multirow{2}{*}{$\begin{array}{l}\text { Renal } \\
\text { Profile }\end{array}$} & \multicolumn{2}{|c|}{ Group 1} & \multicolumn{2}{|c|}{ Group 2} \\
\hline & & $\mathbf{F}$ & Sig. & $\mathbf{F}$ & Sig. \\
\hline \multirow{6}{*}{ Diagnosis } & Sodium & 0.875 & 0.543 & 0.784 & 0.541 \\
\hline & Potassium & 0.967 & 0.471 & 0.609 & 0.658 \\
\hline & Urea & 0.379 & 0.928 & 0.501 & 0.735 \\
\hline & Creatinine & 1.035 & 0.42 & 0.282 & 0.888 \\
\hline & eGFR & 1.666 & 0.126 & 0.657 & 0.625 \\
\hline & Glucose & 0.413 & 0.909 & 0.189 & 0.943 \\
\hline \multirow{6}{*}{$\begin{array}{l}\text { Vascular risk } \\
\text { factors }\end{array}$} & Sodium & 0.045 & 0.833 & 0.001 & 0.981 \\
\hline & Potassium & 0.043 & 0.837 & 0.76 & 0.387 \\
\hline & Urea & 0.044 & 0.835 & 1.542 & 0.22 \\
\hline & Creatinine & 0.02 & 0.889 & 0.591 & 0.445 \\
\hline & eGFR & 0.131 & 0.718 & 2.09 & 0.154 \\
\hline & Glucose & 0.24 & 0.626 & 0.006 & 0.941 \\
\hline \multirow{6}{*}{$\begin{array}{l}\text { Diagnosis* } \\
\text { Vascular risk } \\
\text { factors }\end{array}$} & Sodium & 0.241 & 0.914 & 0.36 & 0.551 \\
\hline & Potassium & 0.365 & 0.832 & 0.001 & 0.974 \\
\hline & Urea & 0.057 & 0.994 & 0.039 & 0.845 \\
\hline & Creatinine & 0.425 & 0.79 & 0.021 & 0.884 \\
\hline & eGFR & 0.279 & 0.891 & 1.494 & 0.227 \\
\hline & Glucose & 0.756 & 0.558 & 0.121 & 0.73 \\
\hline
\end{tabular}

Univariate Analysis of Variance yielded no significant difference between the diagnosis and brain scan results for group 1, F (1, $69)=2.908, p=0.093$ and group $2, F(1,55)=0.284, p=0.596$. Multivariate analysis (MANOVA; table 3) yielded no significant association between both the diagnosis and the presence of vascular risk factors in both groups and the renal profile. There was no significant interaction between diagnosis and vascular risk factors (Table 3).

\section{Discussion}

The link between physical and mental health has long been recognized. More recently, efforts have been made by the Royal college of Psychiatrists to develop a vision for 'parity of esteem' whereby mental and physical health is equally valued [15]. More specifically, the College emphasizes the importance of equal status in the measurement of health outcomes among mental health patients as well as equal efforts to improve quality of care. [15]. Patients with a diagnosis of dementia are recognized as a vulnerable group with multiple co-morbidities.

Within the Welsh NHS, the '1000 lives' national improvement service has focused on dementia as a key area to improve the quality of care [16]. Furthermore, the strategy for older people in Wales (2013 to 2023) outlines the importance of addressing the social, economic and environmental factors that influence health. Vascular dementia in particular is heavily influenced by such factors [17].

It has long been recognized that vascular risk factors have an association with cognitive impairment [17]. For example, Manolio et al and Shilprak et al linked cerebrovascular events with renal insufficiency [18,19]. Seliger et al in 2004 found that moderate renal impairment was associated with a $37 \%$ increased risk of dementia [20].

More recently Bugnicourt et al contrived from epidemiological data to suggest that individuals at all stages of CKD have a higher risk of developing cognitive disorders and dementia [21]. They attribute this link to vascular lesions as well as considering the impact of uraemic toxins causing direct neuronal injury.

Although, in this study, the diagnoses were of different dementia types, a high percentage of the patient group had vascular risk factors. Thus said, moderation of vascular risk factors is relevant in all patients, not just those with vascular dementia. Presence of vascular disease can lead to systemic end organ failure, be that in the brain, kidneys or other. Both kidneys and brain have a similar low vascular resistance system. This allows continuous highvolume perfusion, but also means that both organs are vulnerable to microvascular injury caused by hypertension and diabetes $[22,23]$. These processes of disease would suggest that a strong link would be seen within populations with vascular dementia and CKD.

Using statistical analysis, we compared the cases of dementia/ delirium in different settings with renal biochemical indicators, such as eGFR, urea, and creatinine. There was no significant relationship between the diagnosis and any blood results. It is probable that our results were affected by our sample size. This limited the reliability of our study and further data collection from a larger population group may well show a stronger relationship between different types of dementia/delirium and renal disease. 
Our research highlighted the need to ensure that cognitive testing and brain scans are to be completed for all patients as much as possible. The 1000 Lives campaign made recommendations for assessment process of memory impaired individuals. Within this guidance memory clinics should provide neuropsychological assessment, brain scanning and a physical health screen [24]. NICESCIE recommends a neuropsychological assessment in all cases of suspected dementia. Their guidance recommends that, "assessment should include evaluation of memory, attention, language, praxis, executive function and perception" [25]. In our study population, about half of the patients on the psychiatric unit received this level of assessment. Also, less than one third of the patients had urine infection which could contribute to their confusion and behavioural problems.

\section{Conclusion and Recommendations}

Despite the fact that we found statistically insignificant results our findings highlight need for the multidisciplinary teams to ensure that a proper standardized cognitive assessment such as ACE-III should be done for all patients undergoing diagnosis of dementia. Both mental health directorate and DGH to consider implementing a dementia-physical health pathway. For this, further work is required. Pathway should include collaborative work with other specialties (geriatric, medical, renal directorate) and methods for reducing risk of end organ damage, such as kidney failure in patients with dementia. It's also recommended to repeat the study in the future, on a higher sample size to try to find out the relationship between different types of dementia/delirium and renal disease?

\section{Acknowledgement}

None.

\section{Conflict of Interests}

No conflict of interests.

\section{References}

1. Sun Y, Chen Y, Chen X, Wang J, Xi C, et al. (2009) Change of glomerular rate in healthy adults with aging. Nephrology 14(5): 506-513.

2. Japanese (2007) Clinical Practice Guidebook for Diagnosis and Treatment of Chronic Kidney Disease. Japanese Society of Nephrology 49(7): 757-861.

3. Ninomiya T, Kiyohara Y, Kubo M, Tanizaki Y, Doi Y, et al. (2005) Chronic kidney disease and cardiovascular disease in a general Japanese population: The Hisayama Study. Kidney Int 68(1): 228-236.

4. Ninomiya T, KiyoharaY, Kubo M, Yonemoto K, Tanizaki Y, et al. (2006) Metabolic syndrome and CKD in a general Japanese population: The Hisayama Study. Am J Kidney Dis 48(3): 383-391.

5. Abramson JL, Jurkovitz CT, Vaccarino V, Weintraub WS, Mc Clellan W (2003) Chronic kidney disease, anemia, and incident stroke in amiddle- aged, community-based population: The ARIC Study. Kidney Int 64(2): 610-615.

6. Iseki K (2008) Chronic kidney disease in Japan. Intern Med 47(2): 681689.

7. Imai E, Hori M, Iseki K Yamagata K, Watanabe T, et al. (2007) Prevalence of chronic kidney disease (CKD) in Japanese general population predicted by the MDRD equation modified by a Japanese coefficient. Clin Exp Nephrol 11(2):156-163.

8. Zhang QL, Rothenbacher D (2008) Prevalence of chronic kidney disease in population-based studies: Systematic review. BMC Public Health 8: $117-129$

9. Tamura MK, Wadley V, Yaffe K, Mc Clure LA, Howard G, et al. (2008) Kidney function and cognitive impairment in US adults: The Reasons for Geographic and Racial Differences in Stroke (REGARDS) Study. Am J Kidney Dis 52(2): 227-234.

10. Yaffe K, Ackerson L, Kurella M, Le Blanc P, Kusek JW, et al. (2010) Chronic kidney disease and cognitive function in older adults: Findings from the chronic renal insufficiency cohort cognitive study. J Am Geriatr Soc 58(2): 338-345.

11. Buchman AS, Tanne D, Boyle PA, Shah RC, Leurgans SE et al. (2009) Kidney function is associated with the rate of cognitive decline in the elderly. Neurology 73(12): 920-927.

12. Seliger SL, Siscovick DS, Stehman-Breen CO, Gillen DL, Fitzpatrick A, et al. (2004) Moderate renal impairment and risk of dementia among older adults: The Cardiovascular Health Cognition Study. J Am Soc Nephrol 15(17): 1904-1911

13. Khatri M, Nickolas T, Moon YP, Paik MC, Rundek T, et al. (2009) CKD associates with cognitive decline. J Am Soc Nephrol 20(11): 2427-2432.

14. Etgen T, Sander D, Chonchol M, Briesenick C, Poppert H, et al. (2009) Chronic kidney disease is associated with incident cognitive impairment in the elderly: The INVADE study. Nephrol Dial Transplant 24(10): 3144-3150.

15. Mental health.

16. Mental Health - Improving Dementia Care.

17. Older People.

18. Manolio TA, Kronmal RA, Burke GL, O Leary DH, Price TR (1996) Shortterm predictors of incident stroke in older adults. The Cardiovascular Health Study. Stroke 27(9): 1479-1486.

19. Shlipak MG, Simon JA, Grady D, Lin F, Wenger NK, et al. (2001) Renal insufficiency and cardiovascular events in postmenopausal women with coronary heart disease. J Am Coll Cardiol 38(3): 705-711.

20. Seliger SL, Siscovick, DS, Stehman Breen CO, Gillen DL, Fitzpatrick A, et al. (2004) Moderate renal impairment and risk of dementia among older adults: The Cardiovascular Health Cognition Study. Journal of the American Society of Nephrology 15(7): 1904-1911.

21. O Rourke MF, Safar ME (2005) Relationship between aortic stiffening and microvascular disease in brain and kidney: cause and logic of therapy. Hypertension 46(1): 200-204.

22. (2015) Kidney disease as a determinant of cognitive decline and dementia. Thorleif Etgen Alzheimer's Research \& Therapy 7:29

23. (2010) 1000 Lives Plus. Improving Dementia Care. How to guideline 15.

24. NICE-SCIE (2006). Dementia: supporting people with dementia and their carers in health and social care: Clinical Guideline 42. London, National Institute for Health and Clinical Excellence. 